J. QIAO

KODAI MATH. J.

12 (1989), 429-436

\title{
THE VALUE DISTRIBUTION OF ENTIRE FUNCTIONS OF FINITE ORDER
}

\author{
BY JIAN-YONG QIAO
}

\section{Introduction.}

In [8] Tsuzki proved the following

THEOREM A. Let $f(z)$ be an entire function of order less than one and let $\left\{w_{n}\right\}$ be an unbounded sequence. Assume that there exists a real number $\beta$ such that $0<\beta<\pi / 2$ and all the roots of equations

$$
f(z)=w_{n} \quad(n=1,2, \cdots)
$$

belong to the sector $\{z ;|\arg z-\pi| \leqq \beta\}$. Then $f(z)$ is a linear function.

In [4], [5], [1] Kimura, Kobayashi, Baker and Liverpool improved the above result respectively. In this paper we generalize Theorem $A$ to the following

THEOREM 1. Let $f(z)$ be an entire function and let $\left\{w_{n}\right\}$ be an unbounded sequence. Suppose that for some positive integer $m$.

$$
\lim _{r \rightarrow \infty} \frac{T(r, f)}{r^{m}}=0 \text {. }
$$

Assume that there exists some $\varepsilon>0$ such that all the roots of equations (1) belong to the following set

$$
\bigcup_{k=0}^{m-1}\left\{z ; \frac{2 k}{m} \pi+\varepsilon<\arg z<\frac{2 k+1}{m} \pi-\varepsilon\right\} .
$$

Then $f(z)$ is a polynomial.

The direction $\arg z=\theta$ is said to be a limiting direction of the complex set $E$, if $\theta$ is a cluster point of the set $\{\arg z ; z \in E\}$. As the corollary of Theorem 1 we have

COROllary 1. Let $f(z)$ be an entire function of finite order and let $\left\{w_{n}\right\}$

Received September 7, 1988; Revised March 22, 1989. 
be an unbounded sequence. Assume that $\bigcup_{n=1}^{\infty}\left\{z ; f(z)=w_{n}\right\}$ has only $k(<\infty)$ distinct limiting directions, then $f(z)$ is a polynomial of degree at most $k$.

In [7] Ozawa proposed the following conjecture:

Let $f(z)$ be an entire function, $\left\{w_{n}\right\}$ be an unbounded sequence and $L_{1}, L_{2}$, $\cdots, L_{p}$ be $p$ distinct straightlines any two of which are not parallel with each other. Assume that all the roots of equations (1) lie on $L_{1}, L_{2}, \cdots, L_{p}$. Then $f(z)$ is a polynomial of degree at most $2 p$.

By Corollary 1 we deduce the following

COROLLARY 2. Ozawa's conjecture is true.

A meromorphic function $F(z)$ is said to have a factorization with left factor $f$ and right factor $g$, if it is expressible in the form $f(g(z))$, where $f$ is meromorphic and $g$ is entire ( $g$ may be meromorphic when $f$ is rational). $F(z)$ is said to be pseudoprime if every factorization of the above form implies that either $f$ is rational or $g$ is a polynomial. If $F(z)$ is pseudoprime when only entire factors are considered in the factorization of the above form, it is called $E$-pseudoprime. In this paper we prove the following

THEOREM 2. Let $F(z)$ be a meromorphic function of order less than $m$ (a positive integer). Assume that there exist two complex number $A_{1}, A_{2}$ (finite or infinite) such that all the roots of equation $F(z)=A_{0}(j=1,2)$ belong to the following set

$$
T_{\jmath}=\bigcup_{k=0}^{m-1}\left\{z ; \frac{2 k}{m} \pi+\varepsilon<\arg z-\alpha_{j}<\frac{2 k+1}{m} \pi-\varepsilon\right\}
$$

for some $\varepsilon>0$ and two real numbers $\alpha_{\jmath}(j=1,2)$. Then $F(z)$ is pseudoprime.

In [2] Baker proved the following

THEOREM B. Let $F(z)$ be an entire function of finite order and let there exist a complex number $A$ such that the set of the roots of $F(z)=A$ has only one limiting direction. Then $F(z)$ is E-pseudoprime.

A a corollary of Theorem 2 we improve Theorem $B$ to the following

COROLlaRY 3. Let $F(z)$ be a meromorphic function of finite order and let there exist two distinct complex numbers $A_{1}, A_{2}$ (finite or infinite) such that the set of the roots of $F(z)=A_{j}(j=1,2)$ has only finitely many limiting directions. Then $F(z)$ is pseudoprime.

Let $f(z)$ be an entire function and $f_{1}(z)=f(z), f_{2}(z)=f(f(z)), \cdots, f_{n}(z), \cdots$ be its sequence of interates. Regarding the Fatou set $F(f)$ of those points of 
the complex plane where $\left\{f_{n}(z)\right\}$ does not form a normal family, Baker proved in [2] the following

THEOREM C. Let $f(z)$ be a transcendental entire function and let the set

$$
F(f)-\{z ;|\arg z|<\delta\}
$$

be bounded for every $\delta>0$, then $f(z)$ is of infinite order.

In this paper we improve Theorem $\mathrm{C}$ to the following

THEOREM 3. Let $f(z)$ be a transcendental entire function and let $\theta_{j}(j=1$, $2, \cdots, m)$ be $m$ real numbers. Assume that the set

$$
F(f)-\bigcup_{j=1}^{m}\left\{z ;\left|\arg z-\theta_{j}\right|<\delta\right\}
$$

is bounded for every $\delta>0$, then $f(z)$ is of infinite order.

By Theorem 3 we easily obtain the following

COROLLARY 4. Let $f(z)$ be a transcendental entire function of finite order, then $F(f)$ cannot be contained in any finitely many strip regions.

\section{Some lemmas.}

To prove our theorems, we need the following lemmas.

LEMMA 1. Let $f(z)$ be an entire function with the zeros $\left\{z_{j}\right\}$ and $0<\left|z_{1}\right| \leqq$ $\left|z_{2}\right| \leqq \cdots \leqq\left|z_{j}\right| \leqq \cdots$. Then for any positive integer $n$ we have

$$
\left(\frac{f^{\prime}(z)}{f(z)}\right)^{(n-1)}=(n-1) !\left[-\sum_{|z j| \geqq r} \frac{1}{\left(z_{j}-z\right)^{n}}+O\left(\frac{T(e r, f)}{r^{n}}\right)\right] \quad(r \rightarrow \infty) .
$$

Proof. Let $|z|<r$, by Poisson-Jensen formula we have

$$
\frac{f^{\prime}(z)}{f(z)}=\frac{1}{2 \pi} \int_{0}^{2 \pi} \log \left|f\left(r e^{i \theta}\right)\right| \frac{2 r e^{i \theta}}{\left(r e^{i \theta}-z\right)^{2}} d \theta+\sum_{|z j| \geqq r}\left\{\frac{1}{z-z_{\jmath}}+\frac{\bar{z}_{j}}{r^{2}-\bar{z}_{j} z}\right\} .
$$

Differentiating this $n-1$ times we obtain

$$
\begin{aligned}
\left(\frac{f^{\prime}(z)}{f(z)}\right)^{(n-1)}= & \frac{n !}{2 \pi} \int_{0}^{2 \pi} \log \left|f\left(r e^{i \theta}\right)\right| \frac{2 r e^{i \theta}}{\left(r e^{i \theta}-z\right)^{n+1}} d \theta \\
& +(n-1) ! \sum_{\left|z_{j}\right| \leqslant r}\left\{\frac{(-1)^{n+1}}{\left(z-z_{j}\right)^{n}}+\frac{\bar{z}_{j}^{n}}{\left(r^{2}-\bar{z}_{j} z\right)^{n}}\right\} .
\end{aligned}
$$

We also have 


$$
\begin{gathered}
\left|\frac{1}{2 \pi} \int_{0}^{2 \pi} \log \right| f\left(r e^{i \theta}\right)\left|\frac{2 r e^{i \theta}}{\left(r e^{i \theta}-z\right)^{n+1}} d \theta\right| \leqq O\left(\frac{T(e r, f)}{r^{n}}\right) \quad(r \rightarrow \infty), \\
\left|\sum_{\left|z_{j}\right| \leq r} \frac{\bar{z}_{j}^{n}}{\left(r^{2}-\bar{z}_{j} z\right)^{n}}\right| \leqq O\left(\frac{n(r, f=0)}{r^{n}}\right) \leqq O\left(\frac{T(e r, f)}{r^{n}}\right) \quad(r \rightarrow \infty) .
\end{gathered}
$$

By (6), (7) and (8) we deduce (5), Lemma 1 is thus proved.

LEMMA 2. Let $\theta_{j} \in[0,2 \pi)(j=1,2, \cdots, p)$ be $p$ distinct real numbers. then for any constant $M>0$ there exists some integer $m>M$ such that $\cos m \theta_{0}>\sqrt{3} / 2$ $(j=1,2, \cdots, p)$.

This lemma is Lemma 1.1 of paper [6].

LEMMA 3. If the conditions of Ozawa's conjecture are satisfied, then the order of $f(z)$ is finite.

This lemma is a special case of Theorem 2 of paper [3].

\section{Proof of the theorems.}

Proof of Theorem 1. Let $\omega$ be an $m$-th root of unity. Set

$$
B_{m-j}(z)=(-1)^{\jmath} \sum_{1 \leqq k_{1}<\cdots<k_{j} \leqq m} f\left(\omega^{k_{1}} z\right) f\left(\omega^{k_{2}} z\right) \cdots f\left(\omega^{k} J\right),
$$

$A_{m-j}(z)=B_{m-j}\left(z^{1 / m}\right)$ is obviously an entire function and it is easily seen that

$$
f^{m}(z)+B_{m-1}(z) f^{m-1}(z)+\cdots+B_{1}(z) f(z)+B_{0}(z)=0 .
$$

Thus the entire algebroid function $g(z)=f\left(z^{1 / m}\right)$ satisfies the following equation

Set

$$
g^{m}+A_{m-1}(z) g^{m-1}+\cdots+A_{1}(z) g+A_{0}(z)=0 .
$$

$$
\varphi_{n}(z)=w_{n}^{m}+w_{n}^{m-1} A_{m-1}(z)+\cdots+w_{n} A_{1}(z)+A_{0}(z) .
$$

By (3) it is obvious that the zeros $\left\{a_{n j}\right\}$ of $\varphi_{n}(z)$ (which are the zeros of $g(z)$ $-w_{n}$ ) all lie in the half plane $\operatorname{Im} z>0$.

Because $\left\{w_{n}\right\}$ is unbounded, without loss of generality we may assume that $w_{n} \rightarrow \infty$ as $n \rightarrow \infty$ (otherwise consider its some suitable subsequence). Let $n$ be sufficiently large. It follows from (9) that

$$
\begin{aligned}
\log \varphi_{n}(z) & =m \log w_{n}+\log \left(1+\frac{A_{m-1}(z)}{w_{n}}+\cdots+\frac{A_{0}(z)}{w_{n}^{m}}\right) \\
& =m \log w_{n}+\sum_{j=1}^{\infty} \frac{(-1)^{\jmath-1}}{j}\left(\frac{A_{m-1}(z)}{w_{n}}+\cdots+\frac{A_{0}(z)}{w_{n}^{m}}\right)^{\jmath}
\end{aligned}
$$




$$
=m \log w_{n}+\frac{A_{m-1}(z)}{w_{n}}+O\left(\frac{1}{w_{n}^{2}}\right) \quad(n \rightarrow \infty) .
$$

By this we obtain that

$$
\lim _{n \rightarrow \infty} w_{n}\left[\log \varphi_{n}(z)\right]^{(q)}=A_{m-1}{ }^{(q)}(z) \quad(q=1,2, \cdots) .
$$

From (2) and (9) it follows that

$$
\varliminf_{r \rightarrow \infty} \frac{T\left(r, \varphi_{n}\right)}{r}=0 .
$$

By Lemma 1 and (11) we obtain that there exists a sequence $r_{k} \rightarrow \infty$ such that

$$
\left(\log \varphi_{n}(z)\right)^{\prime}=-\lim _{k \rightarrow \infty} \sum_{\left|a_{n j \mid}\right| r_{k}} \frac{1}{a_{n j}-z} .
$$

Taking $z_{0} \in\{z ; \operatorname{Im} z<0\}$ such that $\varphi_{n}\left(z_{0}\right) \neq 0$, by (12) we deduce that

$$
\lim _{k \rightarrow \infty} \sum_{\mid a} \sum_{n j \mid r_{k}} \frac{\operatorname{Im}\left(a_{n j}-z_{0}\right)}{\left|a_{n j}-z_{0}\right|^{2}}
$$

is a finite number. Since $a_{n j} \in\{z ; \operatorname{Im}(z)>0\}$, we have $\operatorname{Im}\left(a_{n_{j}}-z_{0}\right)>\left|\operatorname{Im}\left(z_{0}\right)\right|>0$. From this we know that the following series is convergent

$$
\sum_{j=1}^{\infty} \frac{1}{\left|a_{n j}-z_{0}\right|^{2}} \text {. }
$$

It tells us that the order of $N\left(r, g\left(z+z_{0}\right)=w_{n}\right)$ is not larger than 2 for every $w_{n}$. By the second fundamental theorem of algebroid functions we obtain that the order of $g(z)$ is not larger than 2. This implies that the order of $\varphi_{n}(z)$ is not larger than 2. By Lemma 1 we have

$$
\left(\log \varphi_{n}(z)\right)^{(q)}=-(q-1) ! \sum_{j=1}^{\infty} \frac{1}{\left(a_{n j}-z\right)^{q}} \quad(q \geqq 3) .
$$

By (10), (12) and (13) we have

$$
\begin{gathered}
A_{m-1}^{\prime}\left(z_{0}\right)=-\lim _{n \rightarrow \infty} w_{n}\left(\lim _{k \rightarrow \infty} \sum_{\left|a_{n j}\right| \leqq r_{k}} \frac{1}{a_{n j}-z_{0}}\right), \\
A_{m-1}^{(q)}\left(z_{0}\right)=-(q-1) ! \lim _{n \rightarrow \infty} w_{n} \sum_{j=1}^{\infty} \frac{1}{\left(a_{n j}-z_{0}\right)^{q}} \quad(q \geqq 3) .
\end{gathered}
$$

By (14) we obtain that

$$
\lim _{n \rightarrow \infty}\left|w_{n}\right| \sum_{j=1}^{\infty} \frac{1}{\left|a_{n j}-z_{0}\right|^{2}} \leqq \frac{\left|A_{m-1}^{\prime}\left(z_{0}\right)\right|}{\left|\operatorname{Im} z_{0}\right|} .
$$

Without loss of generality we may assume that

$$
0<\left|a_{n 1}-z_{0}\right| \leqq\left|a_{n 2}-z_{0}\right| \leqq \cdots \leqq\left|a_{n j}-z_{0}\right| \leqq \cdots .
$$

By (14), (15) and (16) we deduce that for $q>2$ 


$$
\begin{aligned}
\left|A_{m-1}^{(q)}\left(z_{0}\right)\right| & \leqq(q-1) ! \lim _{n \rightarrow \infty}\left|w_{n}\right| \sum_{j=1}^{\infty} \frac{1}{\left|a_{n j}-z_{0}\right|^{q}} \\
& \leqq(q-1) ! \lim _{n \rightarrow \infty} \frac{\left|w_{n}\right|}{\left|a_{n 1}-z_{0}\right|^{q-2}} \sum_{j=1}^{\infty} \frac{1}{\left|a_{n j}-z_{0}\right|^{2}} \\
& \leqq \frac{(q-1) !\left|A_{m-1}^{\prime}\left(z_{0}\right)\right|}{\left|\operatorname{Im}\left(z_{0}\right)\right|} \lim _{n \rightarrow \infty} \frac{1}{\left|a_{n j}-z_{0}\right|^{q-2}} .
\end{aligned}
$$

Since $f\left(a_{n_{1}}^{1 / m}\right)=w_{n}$ and $w_{n} \rightarrow \infty$ as $n \rightarrow \infty$, we have $a_{n 1} \rightarrow \infty$ as $n \rightarrow \infty$. By (17) we deduce that

$$
A_{m-1}^{(q)}\left(z_{0}\right)=0 \quad(q \geqq 3) .
$$

This proves that $A_{m-1}(z)$ is a polynomial of degree at most two. Thus $B_{m-1}(z)$ is a polynomial of degree at most $2 m$. Since

$$
-B_{m-1}(z)=f(\omega z)+f\left(\omega^{2} z\right)+\cdots+f\left(\omega^{m} z\right) .
$$

We easily obtain that $f^{(3 m)}(0)=0$.

For any complex number $c$, set $f_{1}(z)=f(z+c)$. Since all the roots of equations $f(z)=w_{n}(n=1,2, \cdots)$ belong to the set $(3)$, we can easily see that there exists a positive integer $N$ such that all the roots of equations $f_{1}(z)=w_{n}(n=1$, $2, \cdots)$ belong to the following set

$$
\bigcup_{k=0}^{m-1}\left\{z ; \frac{2 k}{m} \pi+\frac{\varepsilon}{2}<\arg z<\frac{2 k+1}{m} \pi-\frac{\varepsilon}{2}\right\}
$$

for any $n>N$. Since $f_{1}(z)$ satisfies all the conditions of $f(z)$, by the above discussion we have $f_{1}^{(3 m)}(0)=0$. Hence $f^{(3 m)}(c)=0$ for any complex number $c$. This proves that $f(z)$ is a polynomial. The proof of Theorem 1 is now complete.

Proof of Corollary 1. Set $\arg z=\theta,(j=1,2, \cdots, k)$ are the limiting directions of $\bigcup_{n=1}^{\infty}\left\{z ; f(z)=w_{n}\right\}$. By Lemma 2 there exists a positive integer $m>\boldsymbol{\rho}_{f}$ (the order of $f(z))$ such that $\cos m \theta_{\jmath}>\sqrt{ } \overline{3} / 2(j=1,2, \cdots, k)$. Hence all $e^{\imath\left(\theta_{j}+\pi / 2 m\right)}$ $(j=1,2, \cdots, k)$ belong to

$$
\bigcup_{k=0}^{m-1}\left\{z ; \frac{2 k}{m} \pi+\frac{\pi}{2 m}<\arg z<\frac{2 k+1}{m} \pi-\frac{\pi}{2 m}\right\} .
$$

It is easily seen that $\arg z=\theta_{j}-\pi / 2 m(j=1,2, \cdots, k)$ are the limiting directions of $\bigcup_{n=1}^{\infty}\left\{z ; f\left(e^{-\imath(\pi / 2 m)} z\right)=w_{n}\right\}$. From this we know that there exists a positive integer $N$ such that all the roots of equations $f\left(e^{-\imath(\pi / 2 m)} z\right)=w_{n}$ belong to the set (18) for any $n>N$. By Theorem 1 we deduce that $f(z)$ is a polynomial. Let $f(z)=a_{q} z^{q}+\cdots+a_{0}$. Then the roots of $f(z)=w_{n}$ should be distributed asymptotically as $q$ roots of $a_{q} z^{q}=w_{n}$ for sufficiently large $n$. Hence $q \leqq k$. 
The proof of Corollary 1 is now complete.

Proof of Corollary 2. By Lemma 3 we see that $f(z)$ is of finite order. We easily know that the limiting directions of $\bigcup_{n=1}^{\infty}\left\{z ; f(z)=w_{n}\right\}$ only may be $\arg z$ $=\theta_{1}, \theta_{2}, \cdots, \theta_{2 p}$ which are parallel with $L_{1}, L_{2}, \cdots, L_{p}$ respectively. By Corollary 1 we thus complete the proof of Corollary 2 .

Proof of Theorem 2. Suppose that $F(z)=f(g(z))$, where $f$ is a transcendental meromorphic function and $g$ is a transcendental entire function. If $f(w)-A_{1}$ has infinitely many zeros $\left\{w_{n}\right\}$, then all the roots of $g(z)=w_{n}(n=1,2, \cdots)$ belong to the set $T_{1}$. Because the order of $F(z)$ is less than $m$, we obviously have

$$
\lim _{r \rightarrow \infty} \frac{T(r, g)}{r^{m}}=0
$$

By Theorem 1, $g(z)$ is a polynomial. This is a contradiction. Hence $f(w)-A_{1}$ has only finitely many zeros and so does $f(w)-A_{2}$. Thus

$$
\frac{f(w)-A_{1}}{f(w)-A_{2}}=R(w) e^{h(w)},
$$

where $R(w)$ is rational, $h(w)$ is entire and nonconstant. It gives us the following equality

$$
\frac{F(z)-A_{1}}{F(z)-A_{2}}=R(g(z)) e^{h(g(z))}
$$

By Pólya's theorem we deduce from (19) that $F(z)$ is of infinite order. This is a contradiction. Hence $F(z)$ is pseudoprime. The proof of Theorem 2 is complete.

Proof of Corollary 3. By the same discussion as in the proof of Corollary 1 , we can obtain that $F(z)$ satisfies all the conditions of Theorem 2 for some positive integer $m$. By Theorem 2 we complete the proof of Corollary 3 .

Proof of Theorem 3. Suppose that $f(z)$ is of finite order. We choose a sequence $\left\{w_{n}\right\} \in F(f)$ such that $w_{n} \rightarrow \infty$ as $n \rightarrow \infty$. Since $F(f)-\bigcup_{j=1}^{m}\left\{z ;\left|\arg z-\theta_{j}\right|\right.$ $<\delta\}$ is bounded for any $\delta>0$, and $\bigcup_{n=1}^{\infty}\left\{z ; f(z)=w_{n}\right\} \subset F(f)$, we know that the number of elements of $\bigcup_{n=1}^{\infty}\left\{z ; f(z)=w_{n}\right\}$ which are outside $\bigcup_{j=1}^{m}\left\{z ;\left|\arg z-\theta_{j}\right|<\delta\right\}$ is at most finite. This implies that the limiting directions of the set $\bigcup_{n=1}^{\infty}\{z ; f(z)$ $\left.=w_{n}\right\}$ only may be $\arg z=\theta_{1}, \theta_{2}, \cdots, \theta_{m}$. By Corollary 1 we deduce that $f(z)$ is a polynomial. This is a contradiction, Theorem 3 is now proved.

Corollary 4 is obtained by 'Theorem 3 . 


\section{REFERENCES}

[1] I. N. BAKER AND L.S.O. Liverpool, The value distribution of entire functions of order at most one, Acta Sci. Math. 41 (1979), 3-14.

[2] I. N. BAKER, The value distribution of composite entire function, Acta. Sci. Math. (Szeged) 32 (1971), 87-90.

[3] A. EdRei AND W.H. J. Fuchs, On meromorphic functions with regions free of poles and zeros, Acta Math. 108 (1962), 113-145.

[4] S. Kimura, On the value distribution of entire functions of order less than one, Kodai Math. Sem. Rep. 28 (1976), 28-32.

[5] T. Kobayashi, Distribution of values of entire functions of lower order less than one, Kodai Math. Sem. Rep. 28 (1976), 33-37.

[6] J. Miles, On entire function of infinite order with radially distributed zeros. Pacific J. Math. 81 (1979), 131-156.

[7] M. Ozawa, On the solution of the functional equation $f \circ g=F(z), V$, Kodai Math. Sem. Rep. 20 (1968), 305-313.

[8] M. TsuzKI, On the value distribution of entire functions of order less than one, J. College of Liberal Arts. Saitama Univ., 9 (1974), 1-3.

Department of Mathematics

Huaibei Teachers College

(supported by coal industry)

Huaibei, Anhui Province

P. R. CHINA 